University of Nebraska - Lincoln

DigitalCommons@University of Nebraska - Lincoln

CSE Conference and Workshop Papers

Computer Science and Engineering, Department

2006

\title{
Cross-Layer Analysis of Error Control in Wireless Sensor Networks
}

Mehmet C. Vuran

University of Nebraska-Lincoln, mcvuran@cse.unl.edu

Ian F. Akyildiz

Georgia Institute of Technology

Follow this and additional works at: https://digitalcommons.unl.edu/cseconfwork

Part of the Computer Sciences Commons

Vuran, Mehmet C. and Akyildiz, Ian F., "Cross-Layer Analysis of Error Control in Wireless Sensor Networks" (2006). CSE Conference and Workshop Papers. 152.

https://digitalcommons.unl.edu/cseconfwork/152

This Article is brought to you for free and open access by the Computer Science and Engineering, Department of at DigitalCommons@University of Nebraska - Lincoln. It has been accepted for inclusion in CSE Conference and Workshop Papers by an authorized administrator of DigitalCommons@University of Nebraska - Lincoln. 


\title{
Cross-Layer Analysis of Error Control in Wireless Sensor Networks
}

\author{
Mehmet C. Vuran Ian F. Akyildiz \\ Broadband \& Wireless Networking Laboratory \\ School of Electrical \& Computer Engineering \\ Georgia Institute of Technology, Atlanta, GA 30332 \\ Tel: (404) 894-5141 Fax: (404) 894-7883 \\ Email:\{mcvuran,ian\}@ece.gatech.edu
}

\begin{abstract}
Severe energy constraints and hence the low power communication requirements amplify the significance of the energy efficient and preferably cross-layer error control mechanisms in Wireless Sensor Networks (WSN). In this paper, a crosslayer methodology for the analysis of error control schemes in WSNs is presented such that the effects of multi-hop routing and the broadcast nature of the wireless channel are investigated. More specifically, the cross-layer effects of routing, medium access and physical layers are considered. This analysis enables a comprehensive comparison of forward error correction (FEC) and automatic repeat request (ARQ) in WSNs.

FEC schemes improve the error resiliency compared to ARQ. In a multi-hop network, this improvement can be exploited by reducing the transmit power (transmit power control) or by constructing longer hops (hop length extension), which can be achieved through channel-aware routing protocols. The results of our analysis reveal that for certain FEC codes, the hop length extension decreases both the energy consumption and the endto-end latency subject to a target PER compared to ARQ. Thus, FEC codes can be regarded as an important candidate for delay sensitive traffic in WSNs. On the other hand, transmit power control results in significant savings in energy consumption at the cost of increased latency. Moreover, the cases where ARQ outperforms FEC codes are indicated for various end-to-end distance and target PER values.
\end{abstract}

\section{INTRODUCTION}

Wireless Sensor Networks (WSNs) are characterized by collaborative information transmission from multiple sensor nodes observing a physical phenomenon [1]. Severe energy constraints of battery-powered sensor nodes necessitate energy-efficient communication protocols in order to fulfill application objectives. Moreover, the low power communication constraints of sensor nodes exacerbate the effects of the wireless channel leading to error-prone links. In WSNs, where correlation between sensors can be exploited in terms of aggregation, collaborative source coding, or correlationbased protocols, energy efficient error control is of extreme importance. Since the these techniques aim to reduce the redundancy in the traffic, it is essential for each packet to be transmitted reliably. Moreover, the strict energy consumption requirements, the multihop structure of the WSNs, and the broadcast nature of the wireless channel necessitate a crosslayer investigation of the effects of error control schemes.
In this paper, a cross-layer analysis of error control schemes is presented. More specifically, the effects of multi-hop routing and the broadcast nature of the wireless communication are investigated to derive the equations governing the energy consumption, latency and packet error rate (PER) performance of error control schemes. As a result, a cross layer analysis considering routing, medium access and physical layers is devised. This analysis enables a comprehensive comparison of forward error correction (FEC) and automatic repeat request (ARQ) schemes in WSNs. So far, the performance of FEC codes have been investigated in a point-to-point fashion [8], [9], [11]. To the best of our knowledge this is the first work which considers both the broadcast wireless channel and multihop structure of WSNs with realistic channel models and a 2 dimensional topology. Moreover, a practical comparison of these schemes is provided by considering two major architectures for WSNs, i.e., Mica2 [4] and MicaZ [5] nodes.

Forward error control (FEC) coding improves the error resiliency by sending redundant bits through the wireless channel. Therefore, lower signal to noise ratio (SNR) values can be supported to achieve the same error rate as an uncoded transmission. This advantage has been generally exploited by reducing the transmit power in cellular networks. This technique, which we refer to as transmit power control, improves the capacity of cellular networks by reducing the interference to other users. On the other hand, in multi-hop networks, the advantage of FEC coding can also be exploited by constructing longer hops. We refer to this technique as hop length extension, which can be achieved through channel-aware cross-layer routing protocols. We investigate the tradeoffs between ARQ and FEC schemes in terms of energy consumption, latency and end-to-end PER considering the transmit power control and hop length extension, which are used to exploit FEC codes. It should be emphasized that in this work, we do not propose a new FEC code for WSNs. Rather, we devise a framework to assess the performance of FEC and ARQ schemes. Furthermore, our goal is to indicate the situations where either of the error control schemes should be favored.

The rest of this paper is organized as follows: In Section II, an overview of previous analysis on error control schemes in WSNs is provided. Our approach and the system model for 
cross-layer analysis are explained in Section III. In Section IV, the cross-layer analysis of ARQ and FEC schemes is presented. The numerical evaluations are explained in Section $\mathrm{V}$ along with their implications on the tradeoffs of error control schemes. Finally, the paper is concluded in Section VI.

\section{RELATED WORK}

Although there have been several studies on error control techniques in wireless networks and especially in cellular networks, none of them are directly applicable to the WSNs. Especially the limited energy consumption requirements of WSNs and the low complexity in the sensor hardware necessitate energy efficient error control and prevent high complexity codes to be deployed. Recently, there have been some work that considers the energy consumption analysis of error control techniques in WSNs.

In [13], the energy consumption profile of convolutional codes has been presented based on $\mu$ AMPS architecture. It has been shown that no convolutional code provides better energy efficiency for probability of bit error, $P_{b}>10^{-5}$ than uncoded transmission [13]. Similarly, in [9], the energy efficiency of convolutional codes are compared to the energy efficiency of $\mathrm{BCH}$ codes in a framework to optimize the packet size in WSNs. The results of this work reveal that the $\mathrm{BCH}$ codes outperform the most energy efficient convolutional code by almost \%15. Consequently, we do not consider convolutional codes in our work due to their energy inefficiency.

In [11], an analysis of different modulation schemes and two $\mathrm{BCH}$ codes is presented based on their energy consumption efficiency. However, in this analysis, the energy consumption for transmitting redundant bits is considered as the only overhead of error control coding without considering the decoding energy. Furthermore, a single-hop WSN is considered, which decouples multi-hop routing and the effects of error control codes. The most relevant work on the analysis of error control coding is presented in [6]. In this work, the effect of error control coding on the energy consumption of multihop WSNs is studied. However, this analysis considers a linear topology, where the distances between each hop are fixed and equal. Moreover, for each link, the probability of error is assumed to be the same. Consequently, the fading effects of the wireless channel and the random route construction cannot be captured with the presented framework in [6]. Furthermore, the end-toend latency has never been considered in the context of FEC codes in WSNs before.

\section{ANALYSis APPROACH AND System MODEL}

In our analysis, we consider a network composed of sensor nodes that are distributed according to a 2-D Poisson distribution with density $\rho$. Duty cycle operation is deployed such that each node is active for $\delta$ fraction of the time and is in sleep mode otherwise [2]. Moreover, we consider a monitoring application such that the reporting rates of sensors are low but the messages should be transmitted reliably.

In order to realize hop length extension, we consider a channel-aware routing algorithm. In this algorithm, the next

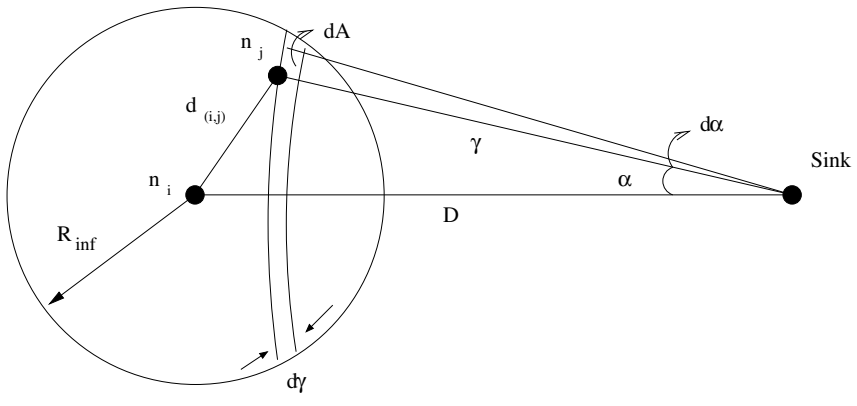

Fig. 1. Reference model for the derivations.

hop is determined according to the received signal to noise ratio (SNR) of a packet sent from a specific node $i$ at a distance $D$ from the sink. Among the neighbors of $i$, the neighbor, $j$, that is closest to the sink and with $\mathrm{SNR}$ value, $\psi_{j}>\psi_{T h}$ is selected as the next hop, where $\psi_{T h}$ is the received SNR threshold. Note that this approach can be implemented using a cross-layer approach as in [2] or through signaling [12]. The medium access is performed through RTS-CTS-DATA exchange in addition to ACK and retransmissions for ARQ.

Accordingly, first, the expected hop distance is derived as a function of the network parameters. Then, the end-to-end energy consumption, latency and PER of a single flow is derived. We use the model shown in Fig. 1 and the log-normal shadow fading channel model [17] for our derivations. Note that in such a model, the transmission range of a node is essentially infinite due to the shadow fading component. In our analysis, we approximate the transmission range of a node to $R_{\text {inf }}$, which is the distance at which the probability that a packet can be successfully received is negligible. Moreover, the hop distance at each hop is considered independent since duty cycle operation is performed. As a result, the state of the network will change at each hop since different nodes will be awake at different time instants. In [15], duty cycle operation is not considered and hence, a different approach has been taken. Note that similar energy consumption analysis has been performed in a node-centric manner for routing algorithms in [14], [16]. However, the effect of neighbor nodes and the effect of routing decisions has not been investigated in this context yet. In our analysis, we investigate the energy consumption to transmit a single packet to the sink with the effect of neighbor nodes and wireless channel effects, which provides a clearer insight into the energy consumption.

Our cross-layer analysis framework enables comprehensive comparison of ARQ and FEC schemes. To illustrate specific results, we consider block codes due to their energy efficiency and lower complexity compared to convolutional codes [13], [9]. We consider a block code, which is represented by $(n, k, t)$, where $n$ is the block length, $k$ is the payload length, and $t$ is the error correcting capability in bits. In our analysis, we use $(128,50,13),(128,78,7)$, and $(128,106,3)$ extended $\mathrm{BCH}$ codes, which enable the evaluation of the effect of error detection capability, $t$, by fixing the block length, $n$. However, other FEC schemes can also be used in our framework. 


\section{CROSS-LAYER ANALYSIS}

In this section, we first derive the expected hop distance by considering the effects of broadcast channel and the routing algorithm discussed in Section III. The results of this analysis are then used to analyze the end-to-end energy consumption, latency and PER of FEC and ARQ schemes. The total energy consumed as a result of a single flow from a source node at distance $D$ from the sink can be found as

$$
E_{\text {flow }}(D)=\mathrm{E}\left[E_{h}\right] \mathrm{E}\left[n_{h}(D)\right]
$$

where $\mathrm{E}\left[E_{h}\right]$ is the expected energy consumption per hop and $\mathrm{E}\left[n_{h}(D)\right]$ is the expected hop count from a source at distance $D$ to the sink. Similarly, the end-to-end latency of a flow is given by

$$
T_{\text {flow }}(D)=\mathrm{E}\left[T_{h}\right] \mathrm{E}\left[n_{h}(D)\right]
$$

where $\mathrm{E}\left[T_{h}\right]$ is the expected delay per hop.

A good approximation for the expected hop count is given in $[16]$ as

$$
\mathrm{E}\left[n_{h}(D)\right] \simeq \frac{D-R_{\text {inf }}}{\mathrm{E}\left[d_{h}\right]}+1
$$

where $\mathrm{E}\left[d_{h}\right]$ is the expected hop distance. In Sections IV-A, IV-B, and IV-C, we derive the expressions for the expected hop length, $\mathrm{E}\left[d_{h}\right]$, the expected energy consumption per hop, $\mathrm{E}\left[E_{h}\right]$, and the expected latency per hop, $\mathrm{E}\left[T_{h}\right]$, respectively.

\section{A. The Expected Hop Distance}

Consider a node $j$ at coordinates $(\gamma, \alpha)$ with respect to the sink as shown in Fig. 1. The distance from node $j$ to node $i$ is, hence, given by

$$
d_{(i, j)}=d(D, \gamma, \alpha)=\sqrt{\gamma^{2}+D^{2}-2 \gamma D \cos \alpha} .
$$

The expected hop distance, $\mathrm{E}\left[d_{h}\right]$, can be found as

$$
\mathrm{E}\left[d_{h}\right]=\int_{\gamma_{\text {min }}}^{D} \int_{-\alpha_{\gamma}}^{\alpha_{\gamma}} d_{(i, j)} d \mathrm{P}\left\{\mathcal{N}_{i}=j\right\}
$$

where $\gamma_{\min }=D-R_{\text {inf }}, d_{(i, j)}$ is the distance between nodes $i$ and $j$ as given by (4), $d \mathrm{P}\left\{\mathcal{N}_{i}=j\right\}$ is the probability that node $j$ is selected as the next hop, and $\alpha_{\gamma}=$ $\operatorname{acos}\left[\left(\gamma^{2}+D^{2}-R_{i n f}^{2}\right) /(2 \gamma D)\right]$.

In order for node $j$ to be selected as the next hop, first, the received SNR, $\psi_{k}$, at each node, $k$, that is closer to the sink than node $j$ should satisfy $\psi_{k}<\psi_{T h}$. Moreover, the received SNR of node $j$ should satisfy, $\psi_{j}>\psi_{T h}$. The probability that node $j$ is selected as the next hop is, hence, given by

$$
\begin{aligned}
d \mathrm{P}\left\{\mathcal{N}_{i}=j\right\}= & \mathrm{P}\left\{N_{A(d \gamma)}=1\right\} \mathrm{P}\left\{\psi_{j}>\psi_{T h}\right\} \\
& \cdot \mathrm{P}\left\{d_{(j, s)} \geq \gamma\right\},
\end{aligned}
$$

where $N_{A(d \gamma)}$ is the number of nodes in the area, $d A$, at distance $\gamma$ from the sink, $\mathrm{P}\left\{\psi_{j}>\psi_{T h}\right\}$ is the probability that the received SNR of a node $j$ is above $\psi_{T h}$, and $\mathrm{P}\left\{d_{(j, s)} \geq \gamma\right\}$ is the probability that the next hop is at least at a distance $\gamma$ from the sink, $s . \mathrm{P}\left\{N_{A(d \gamma)}=1\right\}$ can be approximated by

$$
\begin{aligned}
\mathrm{P}\left\{N_{A(d \gamma)}=1\right\} & \simeq 1-e^{-\rho \delta \gamma d \gamma d \alpha} \text { as } d \gamma \rightarrow 0 \\
& \simeq \rho \delta \gamma d \gamma d \alpha,
\end{aligned}
$$

where we use the approximation $e^{-x} \simeq 1-x$ for the last step since $(\rho \delta \gamma d \gamma d \alpha) \rightarrow 0$ as $d \gamma \rightarrow 0, d \alpha \rightarrow 0$.

For the calculation of $\mathrm{P}\left\{\psi_{j}>\psi_{T h}\right\}$ and $\mathrm{P}\left\{d_{(j, s)} \geq \gamma\right\}$, we first introduce the log-normal channel model [17], where the received power at a receiver at distance $d$ from a transmitter is given by

$$
P_{r}(d)=P_{t}-P L\left(d_{0}\right)-10 \eta \log _{10}\left(\frac{d}{d_{0}}\right)+X_{\sigma},
$$

where $P_{t}$ is the transmit power in $\mathrm{dBm}, P L\left(d_{0}\right)$ is the path loss at a reference distance $d_{0}$ in $\mathrm{dB}, \eta$ is the path loss exponent, and $X_{\sigma}$ is the shadow fading component, with $X_{\sigma} \sim \mathcal{N}(0, \sigma)$. Moreover, the signal to noise ratio (SNR) at the receiver is given by $\psi(d)=P_{r}(d)-P_{n}$ in $\mathrm{dB}$, where $P_{n}$ is the noise power in $\mathrm{dBm}$.

Considering the shadow fading component, $X_{\sigma}$, the probability that $\psi_{j}$, is above some threshold, $\psi_{T h}$, is

$$
\begin{aligned}
\mathrm{P}\left\{\psi_{j}>\psi_{T h}\right\} & =\mathrm{P}\left\{X_{\sigma}>\beta\left(d_{(i, j)}, \psi_{T h}\right)\right\} \\
& =Q\left(\frac{\beta\left(d_{(i, j)}, \psi_{T h}\right)}{\sigma}\right),
\end{aligned}
$$

where

$$
\begin{aligned}
\beta\left(d, \psi_{T h}\right)= & \psi_{T h}+P_{n}-P_{t}+P L\left(d_{0}\right) \\
& +10 \eta \log _{10}\left(\frac{d}{d_{0}}\right)
\end{aligned}
$$

and $Q(x)=1 / \sqrt{2 \pi}\left(\int_{x}^{\infty} e^{-\left(t^{2} / 2\right)}\right) d t$.

According to the channel model above, by denoting the area that consists of nodes that are closer to the sink than node $j$ as $A(\gamma), \mathrm{P}\left\{d_{(j, s)} \geq \gamma\right\}$ can be found as

$$
\begin{aligned}
\mathrm{P}\left\{d_{(j, s)} \geq \gamma\right\} & =\sum_{i=0}^{\infty} \mathrm{P}\left\{N_{A(\gamma)}=i\right\} p_{k}^{i} \\
& =\sum_{i=0}^{\infty} \frac{e^{-M} M^{i}}{i !} p_{k}^{i} \\
& =e^{-M\left(1-p_{k}\right)}
\end{aligned}
$$

where $N_{A(\gamma)}$ is the number of nodes in $A(\gamma), M=\rho \delta A(\gamma)$, and $A(\gamma)$ is the area of intersection of two circles with centers separated by $D$ and with radii $R_{\text {inf }}$ and $\gamma$, respectively. Moreover, $p_{k}=\mathrm{P}\left\{\psi_{k} \leq \psi_{T h}, k \in A(\gamma)\right\}$ is the probability that for a node $k$ in $A(\gamma)$ the received SNR $\psi_{k} \leq \psi_{T h}$, which is given by

$$
p_{k}=\int_{\gamma_{m i n}}^{\gamma} \int_{-\alpha_{\gamma}}^{\alpha_{\gamma}}\left[1-Q\left(\frac{\beta}{\sigma}\right)\right] \frac{1}{A(\gamma)} d \alpha d \gamma .
$$

Using (6), (7), (9), (11), and (12) in (5), the expected hop distance can be calculated as follows:

$$
\mathrm{E}\left[d_{h}\right]=\rho \delta \int_{\gamma_{m i n}}^{D} \int_{-\alpha_{\gamma}}^{\alpha_{\gamma}} \gamma d_{(i, j)} Q\left(\frac{\beta}{\sigma}\right) e^{-M\left(1-p_{k}\right)} d \alpha d \gamma,
$$

which will be used for energy consumption and latency analysis of FEC and ARQ schemes according to (1), (2), and (3). 


\section{B. Energy Consumption Analysis}

The expected energy consumption and latency per hop is also calculated by considering a node $j$ as shown in Fig. 1 . We first derive the expected energy consumption per hop and present the expected latency per hop accordingly. Denoting the expected energy consumption of node $j$ by $\mathrm{E}\left[E_{j}\right]$ and using (13), the expected energy consumption per hop can be calculated as

$$
\mathrm{E}\left[E_{h}\right]=\rho \delta \int_{\gamma_{\min }}^{D} \int_{-\alpha_{\gamma}}^{\alpha_{\gamma}} \gamma \mathrm{E}\left[E_{j}\right] Q\left(\frac{\beta}{\sigma}\right) e^{-M\left(1-p_{k}\right)} d \alpha d \gamma
$$

Since a node can become a next hop if its received SNR value is above a certain threshold, the expected energy consumption, $\mathrm{E}\left[E_{j}\right]$, can be found as

$$
\mathrm{E}\left[E_{j}\right]=\int_{\psi_{T h}}^{\infty} E_{c o m m}\left(\psi, d_{(i, j)}\right) f_{\Psi}\left(\psi, d_{(i, j)}\right) d \psi,
$$

where $E_{c o m m}\left(\psi, d_{(i, j)}\right)$ is the energy consumption for communication between nodes $i$ and $j$ given that they are at a distance $d_{(i, j)}$ and the SNR value at node $j$ is $\psi$. Moreover, $f_{\Psi}(\cdot)$ is the pdf of the SNR. Since, $P(\Psi \leq \psi)=P\left(X_{\sigma} \leq\right.$ $\left.\beta\left(\psi, d_{(i, j)}\right)\right), f_{\Psi}(\cdot)$ is found as:

$$
f_{\Psi}\left(\psi, d_{(i, j)}\right)=f_{X_{\sigma}}\left(\beta\left(\psi, d_{(i, j)}\right)\right)=\frac{1}{\sigma \sqrt{2 \pi}} e^{\frac{-\beta^{2}}{2 \sigma^{2}}} .
$$

The first component, $E_{\text {comm }}\left(\psi, d_{(i, j)}\right)$, in (15) is the energy consumption to transmit a packet between two nodes at a distance $d_{(i, j)}$ with received $\operatorname{SNR} \psi \cdot E_{\text {comm }}\left(\psi, d_{(i, j)}\right)$ has three components as given by ${ }^{1}$

$$
E_{\text {comm }}=E_{T X}+E_{R X}+E_{n e i g h},
$$

where $E_{T X}$ is the energy consumed by the node transmitting the packet (node $i$ ), $E_{R X}$ is the energy consumed by the node receiving the packet (node $j$ ), and $E_{\text {neigh }}$ is the energy consumed by the neighbor nodes.

In order to successfully transmit the packet, a node needs to complete the four-way RTS-CTS-DATA-ACK handshake for ARQ or three-way RTS-CTS-DATA handshake for FEC codes. We denote the probability that a data and a control packet is successfully received at distance $d_{(i, j)}$ by $p_{s}^{D}$ and $p_{s}^{C}$, respectively ${ }^{2}$. Due to the low traffic load, we assume that collisions are avoided through control packets and the probability of RTS collisions is negligible. Accordingly, $E_{T X}$ is given in (18) for ARQ and as follows

$$
E_{T X}^{F E C}=E_{\text {sense }}+E_{t x}^{R}+E_{r x}^{C}+E_{d e c}^{C}+E_{t x}^{D}
$$

for FEC, where $E_{\text {sense }}$ is the energy consumption for sensing the region, $E_{t x}^{x}, E_{r x}^{x}$, and $E_{d e c}^{x}$ are the packet transmission, receiving, and decoding energies for packets, where the superscripts $R, C, D, A$ refer to RTS, CTS, DATA and ACK packets, respectively, and $E_{t / o}$ is the energy consumed

\footnotetext{
${ }^{1}$ We drop the indices $\psi$ and $d_{(i, j)}$ for ease of illustration.

${ }^{2}$ We consider the length of RTS, CTS and ACK packets the same.
}

before timeout. In our calculations, we assume that RTS and CTS packets are also encoded in order to fully exploit the advantages of FEC codes. Using the same approach, the energy consumption of the receiver node is given as follows:

$$
\begin{aligned}
& E_{R X}^{A R Q}=\frac{1}{\left(p_{s}^{C}\right)^{3} p_{s}^{D}}\left\{E_{r x}^{R}+E_{t x}^{C}+E_{r x}^{D}+E_{t x}^{A}\right\} \\
& E_{R X}^{F E C}=E_{r x}^{R}+E_{d e c}^{R}+E_{t x}^{C}+E_{r x}^{D}+E_{d e c}^{D} .
\end{aligned}
$$

The last term in (17), $E_{n e i g h}$, is the energy consumed by the neighbors of the transmitter and the receiver nodes, which is shown in (22) and (23) for ARQ and FEC codes, respectively ${ }^{3}$. Using these derivations in (1), the end-to-end energy consumption can be calculated.

\section{Latency Analysis}

The expression for end-to-end latency of a flow is found using the similar approach above. The delay per hop is given by

$$
\mathrm{E}\left[T_{h}\right]=\rho \delta \int_{\gamma_{\min }}^{D} \int_{-\alpha_{\gamma}}^{\alpha_{\gamma}} \gamma \mathrm{E}\left[T_{j}\right] Q\left(\frac{\beta}{\sigma}\right) e^{-M\left(1-p_{k}\right)} d \alpha d \gamma
$$

where

$$
\mathrm{E}\left[T_{j}\right]=\int_{\psi_{T h}}^{\infty} T_{c o m m}\left(\psi, d_{(i, j)}\right) f_{\Psi}\left(\psi, d_{(i, j)}\right) d \psi,
$$

and $T_{c o m m}$ is given in (26) and (27) for ARQ and FEC, respectively, where $T_{\text {sense }}$ is the time spent for sensing, $T^{C t r l}$ and $T^{D}$ are the control and data packet transmission time, respectively, $T_{t / o}$ is the timeout value, and $T_{d e c}^{C t r l}$ and $T_{d e c}^{D}$ are the decoding latency for control and data packets, respectively.

\section{Decoding Latency and Energy}

The major overhead of FEC codes is the energy consumption for encoding and decoding packets and the delay associated with it. It is well known that the encoding energy for block codes is negligible [8]. Hence, we only consider the decoding energy and latency in our calculations in Sections IV$\mathrm{B}$ and IV-C. The Mica2 and MicaZ nodes that we consider for our analysis do not provide hardware support for FEC coding [4], [5]. Hence, we assume that FEC coding is implemented in software. According to [8], the latency of decoding for a block code $(n, k, t)$ is given as

$$
T_{\text {dec }}^{B L}=\left(2 n t+2 t^{2}\right)\left(T_{a d d}+T_{m u l t}\right),
$$

where $T_{a d d}$ and $T_{m u l t}$ are the energy consumption for addition and multiplication, respectively, of field elements in $\mathrm{GF}\left(2^{m}\right)$, $m=\left\lfloor\log _{2} n+1\right\rfloor[9]$. Both Mica2 and MicaZ nodes are implemented with 8-bit microcontrollers [3], which can perform addition and multiplication of 8 bits in 1 and 2 cycles, respectively. As a result

$$
T_{\text {add }}+T_{\text {mult }}=3\left\lceil\frac{m}{8}\right\rceil t_{\text {cycle }},
$$

\footnotetext{
${ }^{3}$ We assume the header information is sufficient for backoff.
} 


$$
\begin{aligned}
& E_{T X}^{A R Q}=\frac{1}{\left(p_{s}^{C}\right)^{3} p_{s}^{D}}\left\{E_{\text {sense }}+\left(p_{s}^{C}\right)^{2}\left[E_{t x}^{R}+E_{r x}^{C}\right]+\left(1-\left(p_{s}^{C}\right)^{2}\right) E_{t / o}^{C}+\left(p_{s}^{C}\right)^{3} p_{s}^{D}\left(E_{t x}^{D}+E_{r x}^{A}\right)\right. \\
& \left.+\left(p_{s}^{C}\right)^{3}\left(1-p_{s}^{C} p_{s}^{D}\right) E_{t / o}^{A}\right\} \\
& E_{n e i g h}^{A R Q}=\frac{1}{\left(p_{s}^{C}\right)^{3} p_{s}^{D}}\left\{\left(\rho \delta \pi R_{i n f}^{2}-2\right) E_{r x}^{R}+\left[\rho \delta\left(\pi R_{i n f}^{2}-A\left(D, R_{i n f}, D\right)\right)-2\right] E_{r x}^{C}\right\} \\
& E_{n e i g h}^{F E C}=\left(\rho \delta \pi R_{i n f}^{2}-2\right) E_{r x}^{R}+\left[\rho \delta\left(\pi R_{i n f}^{2}-A\left(D, R_{i n f}, D\right)\right)-2\right] E_{r x}^{C} \\
& T_{\text {comm }}^{A R Q}=\frac{1}{\left(p_{s}^{C}\right)^{3} p_{s}^{D}}\left\{T_{\text {sense }}+2\left(p_{s}^{C}\right)^{2} T^{C t r l}+\left(1-\left(p_{s}^{C}\right)^{2}\right) T_{t / o}^{C}+\left(p_{s}^{C}\right)^{3} p_{s}^{D}\left(T^{D}+T^{C t r l}\right)\right. \\
& \left.+\left(p_{s}^{C}\right)^{3}\left(1-p_{s}^{C} p_{s}^{D}\right) T_{t / o}^{A}\right\} \\
& T_{\text {comm }}^{F E C}=T_{\text {sense }}+2 T^{C t r l}+2 T_{d e c}^{C}+T_{t x}^{D}+T_{d e c}^{D}
\end{aligned}
$$

where $t_{\text {cycle }}$ is one cycle duration, which is $250 \mathrm{~ns}$ [3]. Consequently, the decoding energy consumption is $E_{d e c}^{B L}=$ $I_{\text {proc }} V T_{d e c}^{B L}$, where $I_{\text {proc }}$ is the current for processor, and $V$ is the supply voltage.

\section{E. Bit and Packet Error Rate}

In this section, we derive the expressions for bit and packet error rate for Mica2 and MicaZ nodes. Since the modulation schemes used in these nodes are significantly different, it is necessary to investigate the effect of FEC on these nodes separately. Mica2 nodes are implemented with non-coherent FSK modulation scheme. The bit error rate of this scheme is given by [8]

$$
p_{b}^{F S K}=\frac{1}{2} e^{-\frac{E b / N o}{2}}, \quad E b / N o=\psi \frac{B_{N}}{R}
$$

where $\psi$ is the received SNR, $B_{N}$ is the noise bandwidth, and $R$ is the data rate. The modulation scheme used in MicaZ nodes is offset quadrature phase shift keying (O-QPSK) with direct sequence spread spectrum (DSSS). The bit error rate of this scheme is given by [7]

$$
p_{b}^{O Q P S K}=Q\left(\sqrt{(E b / N o)_{D S}}\right)
$$

where

$$
(E b / N o)_{D S}=\frac{2 N \times E b / N o}{N+4 E b / N o(K-1) / 3}
$$

where $N$ is the number of chips per bit, and $K$ is the number of simultaneously transmitting users.

Based on the bit error rate $p_{b}$, the PER for ARQ and FEC codes can be calculated as follows. For ARQ, the CRC-16 error detection mechanism is deployed in both Mica nodes. Assuming all possible errors in a packet can be detected, the PER of a packet with payload $l$ bits is given by

$$
\operatorname{PER}^{C R C}(l)=1-\left(1-p_{b}\right)^{l}
$$

For the $\mathrm{BCH}$ codes considered here, assuming perfect interleaving at the transceiver, the block error rate (BLER) is given by

$$
\operatorname{BLER}(n, k, t)=\sum_{i=t+1}^{n}\left(\begin{array}{l}
n \\
i
\end{array}\right) p_{b}^{i}\left(1-p_{b}\right)^{n-i}
$$

Since a packet can be larger than the block length $n$ especially where small block lengths are used, the PER for FEC is given by

$$
\operatorname{PER}^{F E C}(l, n, k, t)=1-(1-\operatorname{BLER}(n, k, t))^{\left\lceil\frac{l}{k}\right\rceil}
$$

where $\left\lceil\frac{l}{k}\right\rceil$ is the number of blocks required to send $l$ bits and $\lceil\cdot\rceil$ is the ceiling function.

\section{NUMERICAL RESULTS}

In this section, we investigate the effect of FEC scheme in terms of PER, energy consumption and end-to-end latency in a multi-hop network via numerical evaluations in MATLAB. The cases where FEC can be more favorable than ARQ are discussed. Moreover, an energy and latency-based taxonomy is devised to qualitatively compare FEC schemes with ARQ. For this comparison, two sensor node architectures are considered, i.e., Mica2 [4] and MicaZ [5]. We consider a multi-hop network, where a random access scheme and a channel-aware routing protocol is deployed as discussed in Section III. Unless otherwise noted, the parameters in Table I are used for the numerical results. For Mica2 nodes, the experimental values in [10] is used while for MicaZ, the values on the datasheet are used [5].

The expected hop distance $d_{h o p}$, which is found in (5), is shown in Fig. 2 (a) as a function of the received SNR threshold, $\psi_{T h}$, for different transmit power values, $P_{t}$. It can be observed that for small values of the received SNR threshold, $\psi_{T h}$, the average hop distance increases. Since lower $\psi_{T h}$ allows nodes with lower channel quality to be chosen as the next hop, further nodes may become the next hop. Therefore, the number of hops from a node to a sink 


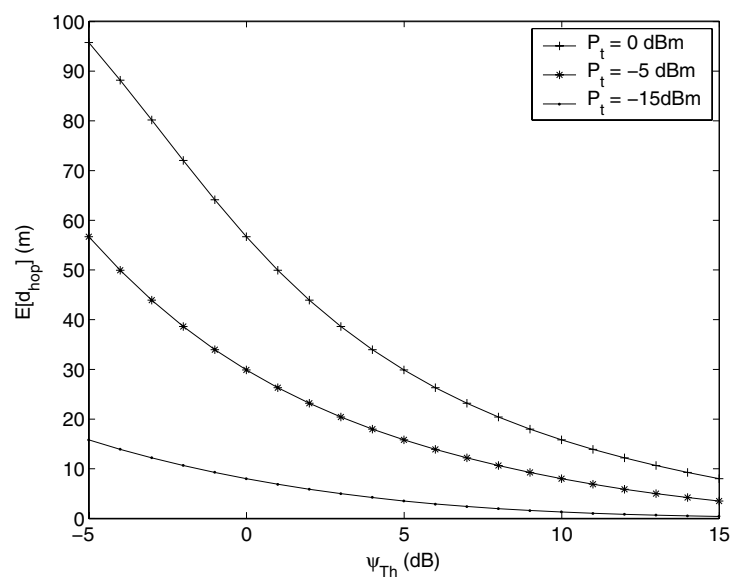

(a)

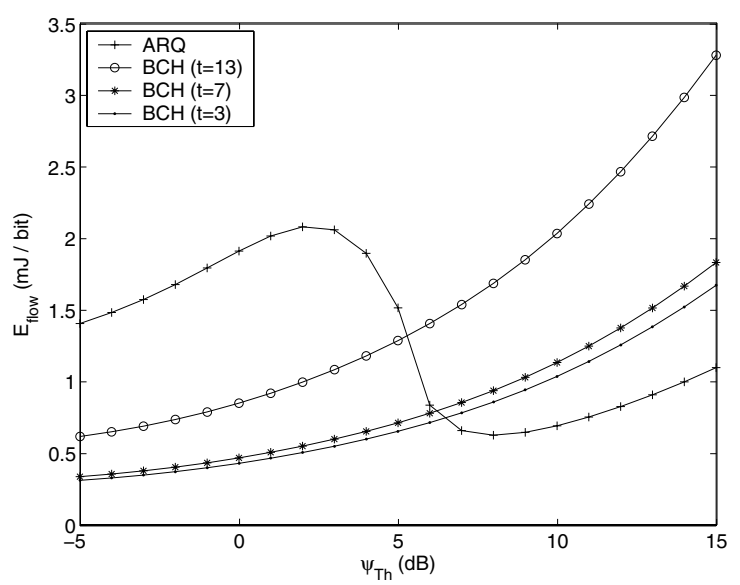

(b)

Fig. 2. (a) Avg. hop distance and (b) avg. energy consumption of a flow vs. $\psi_{T h}$ (DSSS+OQPSK).

TABLE I

PARAMETERS

\begin{tabular}{|l|l||l|l|}
\hline$D$ & $300 \mathrm{~m}$ & $l_{C}$ & 8 bytes \\
$P_{t}$ & $0,-5,-15 \mathrm{dBm}$ & $l_{D}$ & 38 bytes \\
$P L_{d 0}$ & $55 \mathrm{~dB}$ & $t_{c y c l e}$ & $250 \mathrm{~ns}$ \\
$P_{n}$ & $-105 \mathrm{dBm}$ & $I_{\text {proc }}$ & $8 \mathrm{~mA}$ \\
$\eta$ & 3 & $\mathrm{~V}$ & $3 \mathrm{~V}$ \\
$\sigma$ & 3.8 & & \\
\hline & Mica2 & MicaZ \\
\hline$e_{r x}\left(P_{t}=0\right)$ & $21 \mathrm{~mJ}$ & $59.1 \mathrm{~mJ}$ \\
$e_{t x}$ & $52.2 \mathrm{~mJ}$ \\
$e_{t x}\left(P_{t}=-5\right)$ & $21.3 \mathrm{~mJ}$ & $42 \mathrm{~mJ}$ \\
$e_{t x}\left(P_{t}=-15\right)$ & $16.2 \mathrm{~mJ}$ & $29.7 \mathrm{~mJ}$ \\
$t_{b i t}=1 / R$ & $62.4 \mu \mathrm{s}$ & $4 \mu \mathrm{s}$ \\
$N$ & N/A & $16 \mathrm{chips}$ \\
$K$ & N/A & 2 \\
\hline
\end{tabular}

decreases for smaller $\psi_{T h}$ values. Moreover, when the transmit power of a node is decreased, the expected hop distance decreases as expected.

In the following, we present the effects of two techniques to exploit FEC codes in WSNs, i.e., hop length extension and transmit power control defined in Section I. Moreover, the effects of end-to-end distance and the end-to-end target PER on the choice of error control scheme are also discussed.

\section{A. Hop Length Extension}

In Fig. 2 (b), the end-to-end energy consumption per useful bit is shown as a function of the SNR threshold, $\psi_{T h}$. The energy consumption is shown for both ARQ with 7 retransmissions and three different FEC schemes as discussed in Section III. As shown in Fig. 2 (b), the energy consumption of a flow decreases for smaller $\psi_{T h}$ values. This is mainly because of the increase in expected hop distance as shown in Fig. 2 (a). However, the energy consumption for ARQ significantly increases as $\psi_{T h}$ is decreased below a specific value, e.g., 7 $\mathrm{dB}$. A lower $\psi_{T h}$ results in nodes with lower channel quality to be selected as the next hop. As a result, retransmissions occur, which increase the energy consumption per hop. Although the expected number of hops decreases, the increase in energy consumption per hop dominates the total energy consumption for ARQ. Note that for ARQ, the energy consumption curve reaches a peak point and decreases as $\psi_{T h}$ is decreased. This point corresponds to the case that the maximum number of retransmissions is not sufficient for reliable communication. When the FEC codes are considered, the energy consumption is proportional to the error correcting capability, $t$, of the code. Since the code rate is decreased for higher $t$, the energy consumption per useful bit increases. When ARQ and FEC codes are compared, for high $\psi_{T h}$ values, ARQ outperforms the FEC codes. However, the two BCH codes with $t=3$ and $t=7$ are more energy efficient for higher $\psi_{T h}$. Although this figure clearly shows the energy consumption of the two schemes as a function of $\psi_{T h}$, the operating points of $\psi_{T h}$ for ARQ and FEC has to be determined. Hence, next, we investigate the end-to-end error performance.

The PER for CRC and FEC codes are given in (32) and (34), respectively. Since these equations show the PER for a single hop, here we extend these equations for the multi-hop case. Note that, WSN applications are interested in the achievable end-to-end PER bound rather than the single hop PER. Hence, the relation between $\psi_{T h}$ and the end-to-end PER bound can be used to determine the optimal point for $\psi_{T h}$. Denoting the PER of a hop $i$ by $P E R_{i}$, there exists a $\pi$ such that

$$
P E R_{i} \leq \pi, \text { for } \psi_{i} \geq \psi_{T h},
$$

where $\psi_{i}$ is the received SNR of the hop and $\pi=f\left(\psi_{T h}\right)$, which can be calculated using (30) - (34) depending on the modulation scheme, the error control scheme, the channel characteristics, and the packet length. Since the end-to-end PER is

$$
P E R_{e 2 e}=1-\Pi_{i=1}^{n_{h}}\left(1-P E R_{i}\right),
$$

where $n_{h}$ is the number of hops, $P E R_{e 2 e}$ is bounded by

$$
P E R_{e 2 e} \leq 1-(1-\pi)^{n_{h}}, \text { for } \psi_{i} \geq \psi_{T h}, \forall i .
$$




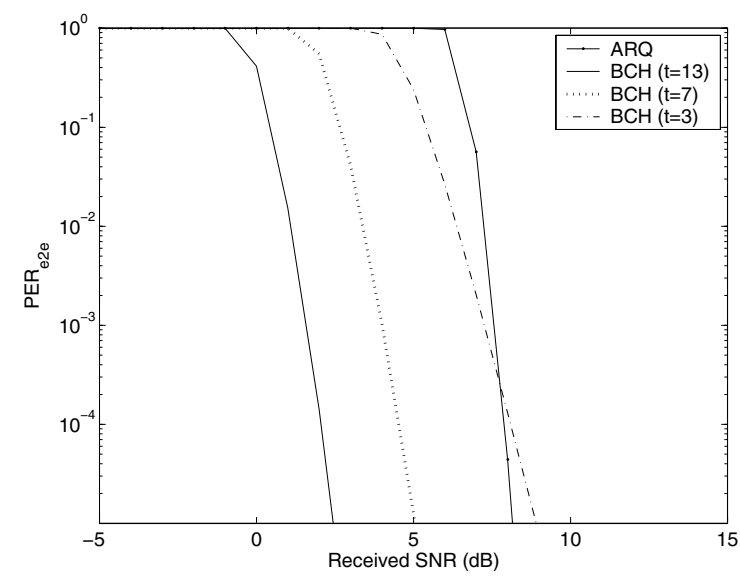

(a)

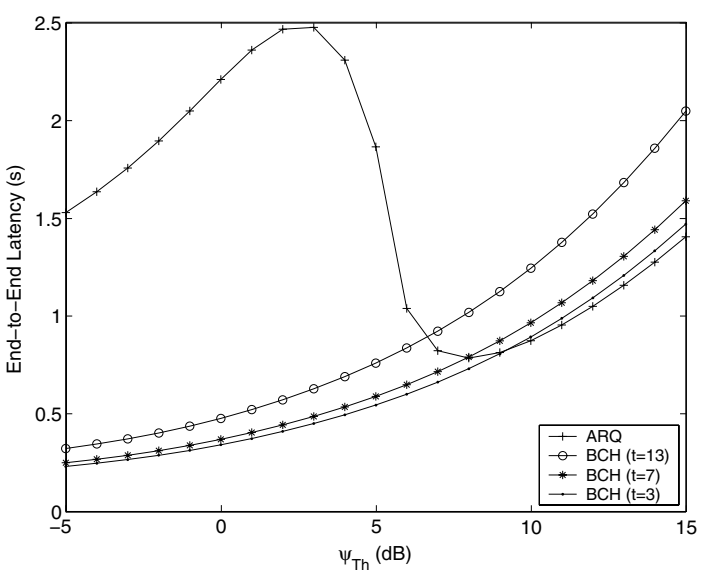

(b)

Fig. 3. (a) End-to-end PER and (b) avg. end-to-end latency vs. $\psi_{T h}$ (DSSS+OQPSK).

Now assume that the end-to-end PER needs to be bounded by a certain threshold, $P E R_{e 2 e}^{*}$, according to a particular application. Accordingly, the route selection needs to be performed such that

$$
\psi_{T h}=f^{-1}\left(1-\left[1-P E R_{e 2 e}^{*}\right]^{1 / n_{h}}\right) .
$$

The relationship between the end-to-end PER, $P E R_{e 2 e}$, and $\psi_{T h}$ is shown in Fig. 3 (a) for MicaZ nodes, where the optimum point for $\psi_{T h}$ can be found. As an example, if the target PER of an application is $10^{-2}$, the minimum value for $\psi_{T h}$ corresponds to $\sim 7 \mathrm{~dB}$ for ARQ and $\sim 3 \mathrm{~dB}$ for $\mathrm{BCH}$ code with $t=7$. As a result, it can be observed from Fig. 2 (b) that $\mathrm{BCH}$ with $t=7$ is slightly more energy efficient than ARQ. It is clear that more energy is consumed per hop for FEC codes due to both transmission of redundant bits and decoding. However, since the effective error rate of the channel is decreased with FEC codes, lower SNR values can be supported. By deploying a routing protocol that exploits this property, longer hop distances can be achieved leading to lower end-to-end energy consumption. However, note that the energy consumption of the $\mathrm{BCH}$ code with $t=13$ is still above the minimum value achieved by ARQ as shown in Fig. 2 (b), which suggests that an optimal value for $t$ should be chosen.

Exploiting FEC schemes with channel-aware routing not only improves energy consumption performance but the endto-end latency can also be decreased significantly as shown in Fig. 3 (b). It is clear that the three FEC schemes outperform ARQ since their optimal $\psi_{T h}$ value is lower than ARQ. This is due to both longer hops for FEC codes and the additional retransmissions of ARQ. Since the decoding delay of the FEC codes is lower than the time consumed for retransmission of a packet, FEC schemes improve the latency performance of the WSNs.

In order to capture the efficiency of an error control scheme, we propose a taxonomy function that consists of the energy consumption, latency and PER performance. This function is given as follows:

$$
\mathcal{T}=\frac{l_{D}}{E_{\text {flow }} T_{\text {flow }}}\left(1-P E R_{\text {e2e }}\right)
$$

where $l_{D}$ is the payload length, $E_{\text {flow }}, T_{\text {flow }}$, and $P E R_{e 2 e}$ are the end-to-end energy consumption, latency and PER, respectively. Note that a similar efficiency function has been proposed in [9], where the energy efficiency and the reliability of a single hop has been considered. In our approach, we also consider the cost for latency and propose a multi-hop taxonomy function.

In Fig. 4 (a) and (b) the taxonomy function is evaluated for MicaZ and Mica2 nodes, respectively. The taxonomy function is normalized for the maximum value of ARQ . It is clear from (37) that a higher value of $\mathcal{T}$ corresponds to higher efficiency. It can be observed from Fig. 4 (a) that for the MicaZ nodes, the FEC codes outperform ARQ. Moreover, an optimal error correction capability, $t$, can be found that leverages the PER with energy consumption and latency. On the other hand, for Mica2 nodes, ARQ is more efficient than the FEC codes. This interesting result advocates that there is no clear winner for error control techniques in WSNs and their performance directly depends on the node hardware.

The reason behind the difference between MicaZ and Mica2 nodes can be explained as follows. In Fig. 4 (c), the taxonomy function is re-evaluated without considering the energy consumption of neighbor nodes, $E_{\text {neigh }}$, in (17). In this case, $\mathrm{BCH}$ codes with $t=3$ and $t=7$ are more efficient than ARQ. The major difference between Mica2 and MicaZ nodes is the data rate of the transceivers and the modulation schemes. As shown in Table I, the time consumed for transmitting a bit is 15 times higher for Mica2 than MicaZ. This corresponds to significant energy consumption for communication. Since the peak of the ARQ curve corresponds to no retransmissions, it is clear that the FEC codes consume more energy primarily due to the transmission of redundant bits. When the receiving energy consumption of the neighbors are considered, the energy consumption significantly increases. Moreover, $\mathrm{BCH}$ 


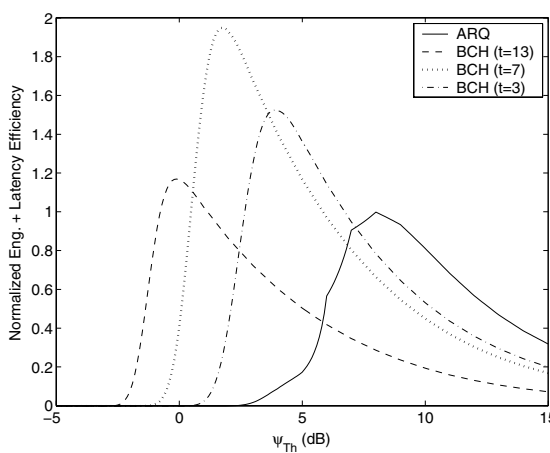

(a)

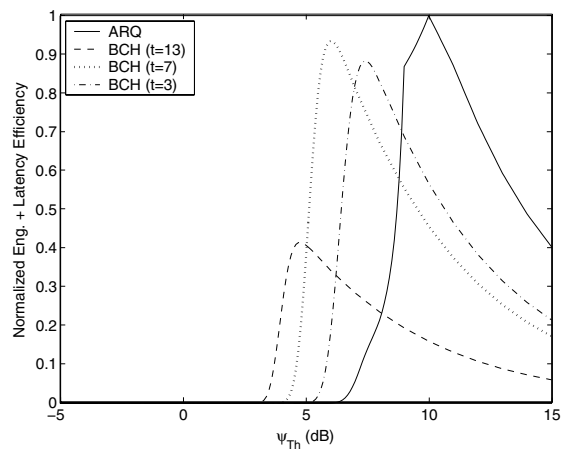

(b)

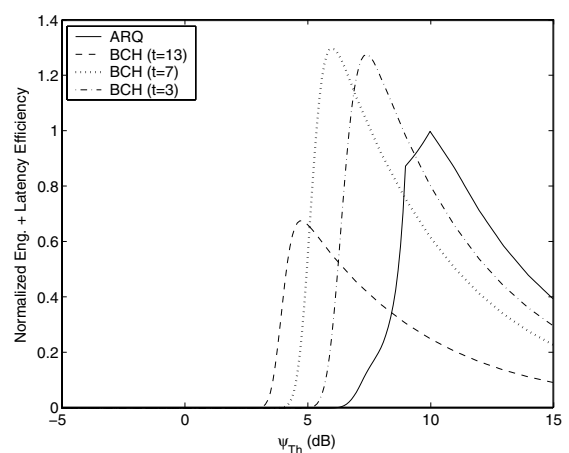

(c)

Fig. 4. Taxonomy function vs. $\psi_{T h}$ for (a) DSSS+OQPSK, (b) FSK, and (c) FSK without $E_{n e i g h}$.

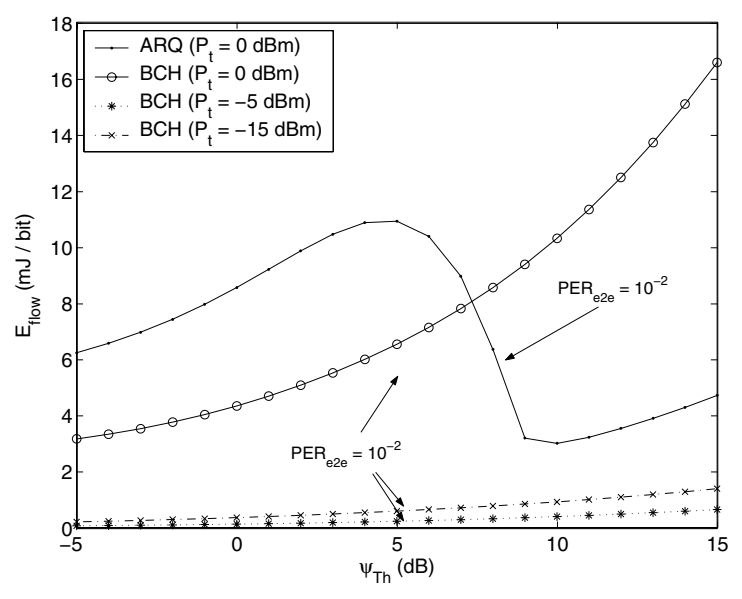

(a)

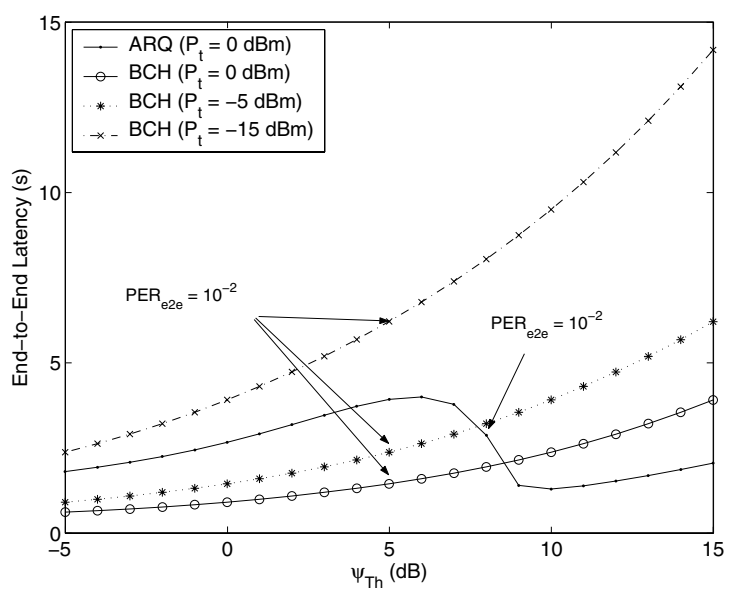

(b)

Fig. 5. (a) Avg. energy consumption of a flow and (b) avg. end-to-end latency vs. $\psi_{T h}$ for different values of transmit power (FSK).

codes lead to smaller increase of expected hop length for Mica2 nodes than MicaZ nodes. As a result, for Mica2 nodes, the advantage of longer hop distance of the FEC codes is outweighed by the increase in energy consumption of neighbor nodes. This favors ARQ for Mica2 nodes and shows that consideration of $E_{n e i g h}$ is important to accurately assess the performance of ARQ and FEC.

\section{B. Transmit Power Control}

Another technique to exploit FEC codes is to match the average hop distance of FEC codes with ARQ. This can be achieved by decreasing the transmit power, $P_{t}$. In order to investigate the effect of transmit power, $P_{t}$, we consider three power levels, i.e., $0,-5$, and $-15 \mathrm{dBm}$ supported by both Mica2 and MicaZ. Intuitively, decreasing transmit power can improve the energy efficiency of the FEC schemes, since less power is consumed for transmission of longer encoded packets. Although the receive power is fixed, since the interference range of a node decreases, the number of neighbors that consume idle energy also decreases. On the other hand, decreasing transmit power increases the number of hops. In Fig. 5 (a), the energy consumption of $\mathrm{BCH}$ code with $t=13$ is shown for three different transmit power levels and ARQ at $P_{t}=0 \mathrm{dBm}$. Furthermore, the operating points of these two schemes corresponding to $P E R_{e 2 e} \leq 10^{-2}$ are indicated. Note that the decrease in transmit power decreases the endto-end energy consumption outperforming ARQ. However, the drawback of the transmit power control is shown in Fig. 5 (b), where the end-to-end latency is shown. Contrary to the hop distance extension, since controlling transmit power has no effect in the time required for transmitting a packet, the endto-end latency depends on the number of hops. Since transmit power control increases the number of hops, this technique introduces a tradeoff between energy consumption and latency.

\section{Effects of End-to-End Distance and Target PER}

In this section, we investigate the effects of end-to-end distance, $D$, and the target PER, $P E R_{e 2 e}^{*}$, on the performance of ARQ and FEC schemes. The end-to-end energy consumption per useful bit is shown in Fig. 6 (a) and (b) as a function of the end-to-end distance, $D$, for Mica2 and MicaZ nodes, respectively. In these figures, the minimum $\psi_{T h}$ is selected for each $\mathrm{BCH}$ code such that $P E R_{e 2 e}^{*} \leq 10^{-2}$ is satisfied. For ARQ, two curves are shown. The solid line corresponds to 


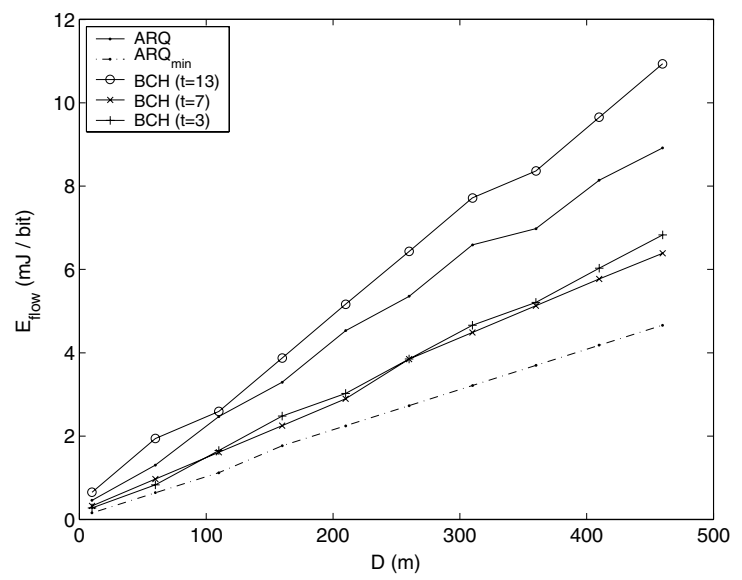

(a)

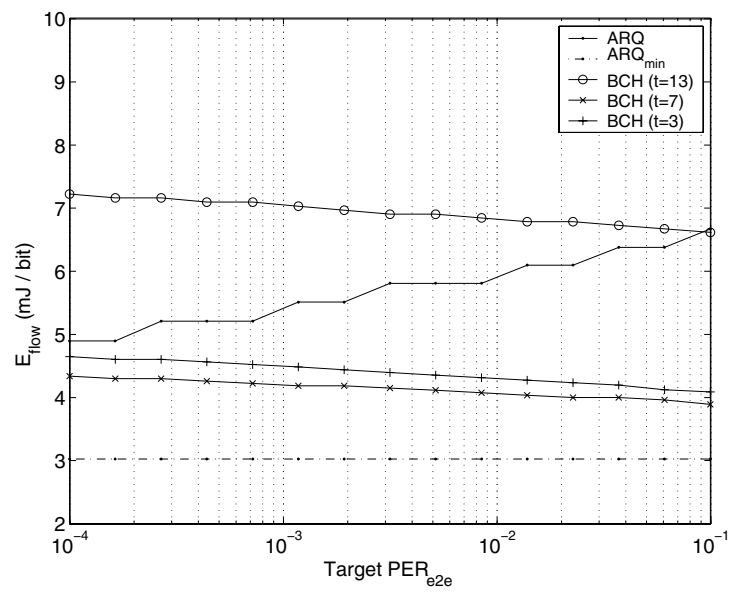

(c)

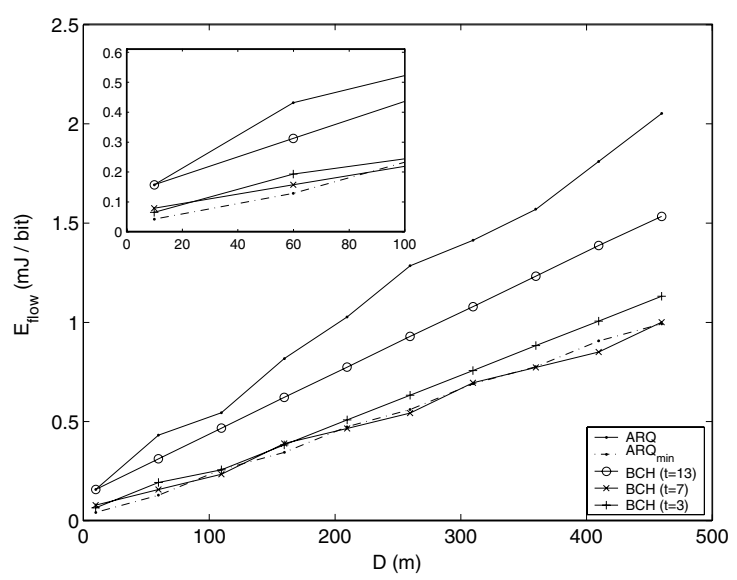

(b)

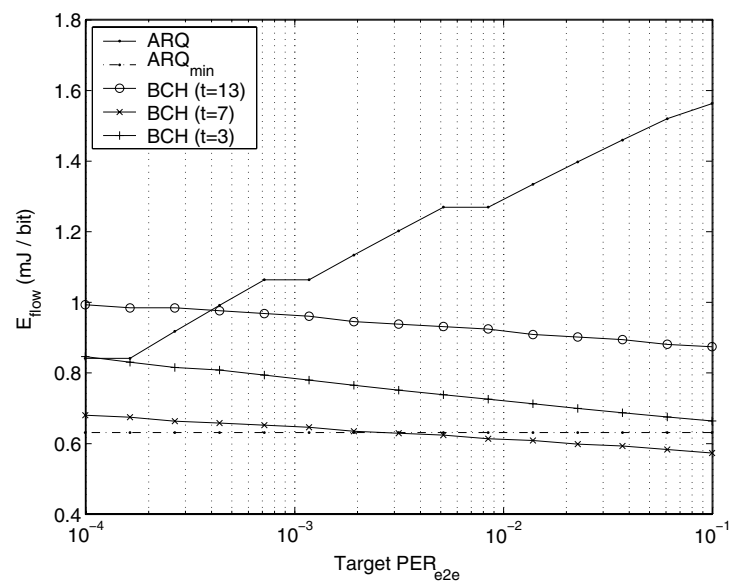

(d)

Fig. 6. Average energy consumption vs. end-to-end distance for (a) Mica2 and (b) MicaZ nodes, and the average energy consumption vs. target end-to-end PER for (c) Mica2 and (d) MicaZ nodes.

the minimum $\psi_{T h}$ that satisfies the PER constraint. However, note from Fig. 5 (a) that the minimum energy consumption of ARQ corresponds to a higher $\psi_{T h}$ value than the one found here. Since the energy consumption of the ARQ is minimum without retransmissions, a higher link quality is required to achieve the optimum performance. Hence, in Figs. 6 (a)-(d), the line denoted by $A R Q_{\min }$ corresponds to $\psi_{T h}$ that achieves the minimum energy consumption for ARQ. Accordingly, for Mica2 nodes, ARQ is more energy efficient than the FEC codes irrespective of the end-to-end distance. On the other hand, for MicaZ nodes, $\mathrm{BCH}$ code with $t=7$ results in energy consumption comparable to ARQ. In particular, the magnified view in Fig. 6 (b) reveals that ARQ is more energy efficient for end-to-end distances up to $80 \mathrm{~m}$, which corresponds to $\sim 5$ hops for ARQ and $\sim 3$ hops for the BCH code with $t=7$. For hop counts higher than these values, the $\mathrm{BCH}$ code consumes slightly less energy compared to ARQ.

As explained before, the operating point of $\psi_{T h}$ is determined according to the target PER of the WSN application. The effect of target PER is investigated in Figs. 6 (c)-(d) for Mica2 and MicaZ nodes, respectively. Similar to our observations above, ARQ is more energy efficient for Mica2 nodes irrespective of the target PER. However, when MicaZ architecture is considered, $\mathrm{BCH}$ code outperforms ARQ for target $P E R_{e 2 e}>0.002$. As the target PER is increased, the optimal value of $\psi_{T h}$ is decreased improving the energy efficiency of FEC codes. As a result, energy consumption of $\mathrm{BCH}$ code with $t=7$ is more favorable than ARQ.

\section{CONCLUSIONS}

In this paper, a cross-layer analysis of error control schemes is presented. Forward error control (FEC) coding improves the error resiliency by sending redundant bits through the wireless channel. It is shown that this improvement can be exploited by transmit power control or hop length extension through channel-aware cross-layer routing protocols in WSNs. The results of our cross-layer analysis is summarized in Table II, where the efficient schemes are identified. Accordingly, hop length extension decreases both energy consumption and endto-end latency for certain FEC codes when compared to ARQ. 
TABLE II

OVERVIEW OF RESULTS

\begin{tabular}{|l|l|l|l|l|}
\hline \multirow{2}{*}{} & \multicolumn{2}{|l|}{ Hop Length Ext. } & \multicolumn{2}{l|}{ Tx. Power Cont. } \\
\cline { 2 - 5 } & Energy & Latency & Energy & Latency \\
\hline Mica2 & ARQ & $\begin{array}{l}\text { BCH } \\
(\mathbf{t} \leq \mathbf{7})\end{array}$ & $\begin{array}{l}\text { BCH } \\
(\mathbf{t} \geq \mathbf{1})\end{array}$ & ARQ \\
\hline MicaZ & $\begin{array}{l}\text { BCH } \\
(\mathbf{t}=\mathbf{7})\end{array}$ & $\begin{array}{l}\text { BCH } \\
(\mathbf{t} \geq \mathbf{1})\end{array}$ & $\begin{array}{l}\text { BCH } \\
(\mathbf{t} \geq \mathbf{1})\end{array}$ & ARQ \\
\hline
\end{tabular}

On the other hand, transmit power control can be exploited in situations where energy consumption is of paramount importance and can be traded off for end-to-end latency. Moreover, it has been shown that the selection of suitable error control scheme depends on the physical architecture of the sensor nodes as well as the end-to-end distance and target PER. Finally, FEC schemes are shown to significantly improve the end-to-end latency performance of WSNs through hop length extension without hampering the energy efficiency and end-toend PER, which make them an important candidate for delay sensitive traffic in WSNs.

\section{ACKNOWLEDGEMENT}

This work is supported by the National Science Foundation under contract CNS-0519841.

\section{REFERENCES}

[1] I. F. Akyildiz, W. Su, Y. Sankarasubramaniam, and E. Cayirci, "Wireless Sensor Networks: A Survey", Computer Networks (Elsevier) Journal, Vol. 38, No. 4, pp. 393-422, March 2002.
[2] I. F. Akyildiz, M. C. Vuran, and O. B. Akan, "A Cross-Layer Protocol for Wireless Sensor Networks," in Proc. Conference on Information Sciences and Systems (CISS '06), Princeton, NJ, March 22-24, 2006.

[3] Atmel Corp. ATmega128 Datasheet. http://www.atmel.com.

[4] Crossbow Corp. Mica2 Datasheet. http://www.xbow.com.

[5] Crossbow Corp. MicaZ Datasheet. http://www.xbow.com.

[6] H. Karvonen, Z. Shelby, C. Pomalaza-Raez, "Coding for energy efficient wireless embedded networks," in Proc. Int. Workshop on Wireless AdHoc Networks, pp. 300 - 304, June 2004.

[7] M. A. Landolsi, W. E. Stark, "On the accuracy of Gaussian approximations in the error analysis of DS-CDMA with OQPSK modulation," IEEE Trans. Comm., vol. 50, no. 12, Dec. 2002.

[8] S. Lin, and D. J. Costello, Jr., "Error control coding: fundamentals and applications," Prentice-Hall, 1983.

[9] Y. Sankarasubramaniam, I. F. Akyildiz, S. W. McLaughlin, "Energy efficiency based packet size optimization in wireless sensor networks," in Proc. IEEE Internal Workshop on Sensor Network Protocols and Applications, pp. 1 -8, 2003.

[10] V. Shnayder, et.al., "Simulating the power consumption of large-scale sensor network applications," in Proc. ACM SenSys'04, Baltimore, MD, November 2004.

[11] K. Schwieger, A. Kumar, G. Fettweis, "On the impact of the physical layer on energy consumption in sensor networks," in Proc. EWSN '05, pp. ; pp. 13 - 24, Feb. 2005.

[12] K. Seada, M. Zuniga, A. Helmy, B. Krishnamachari, "Energy-efficient forwarding strategies for geographic routing in lossy wireless sensor networks,", in Proc. ACM Sensys '04, November 2004.

[13] Shih, E. et al., "Physical layer driven protocol and algorithm design for energy-efficient wireless sensor networks," in Proc. ACM Mobicom 2001, pp. 272-286, Rome, Italy, July 2001.

[14] P. Skraba, H. Aghajan, A. Bahai, "Cross-layer optimization for high density sensor networks: Distributed passive routing Decisions," in Proc. Ad-Hoc Now'04, Vancouver, July 2004.

[15] S. Vural, E. Ekici, "Analysis of hop-distance relationship in spatially random sensor networks," in Proc. ACM MobiHoc '05, pp. 320-331, May 2005.

[16] M. Zorzi, R. Rao, "Geographic random forwarding (GeRaF) for ad hoc and sensor networks: multihop performance," IEEE Trans. Mobile Computing, vol. 2, no. 4, pp. 337-348, Dec. 2003.

[17] M. Zuniga, B. Krishnamachari, "Analyzing the transitional region in low power wireless links," in Proc. IEEE SECON '04, pp. 517 - 526, Oct. 2004. 